\title{
Ciprofloxacin resistance in Salmonella enterica serotype Typhi/ Paratyphi and antimicrobial agent quality and bioavailability
}

The concern about the poor efficacy of fluoroquinolones as the first-line therapy for enteric fever (Renuka et al., 2005) is legitimate, especially for those in endemic areas. To address this problem, surveillance for multidrug-resistant Salmonella enterica serotype Typhi/Paratyphi isolates should include monitoring of the quality and bioavailability of antimicrobials offered to patients.

The results of assays undertaken in Nigeria of the active components of therapeutic agents, including antimicrobials, at pharmacy outlets are astounding. Active ingredients in as many as $48 \%$ of samples of common medicines offered to consumers at pharmacy sites have been found to be outside the limits specified by the British Pharmacopoeia (Taylor et al., 2001). It is likely that a similar problem exists in several other countries where enteric fever is endemic. The possibility of fluoroquinolone resistance amongst Salmonella enterica serotype Typhi/
Paratyphi isolates that are already fully resistant to chloramphenicol, ampicillin and trimethoprim-sulfamethoxazole considerably exacerbates the problem.

The bioavailability of medicines generally, and antibiotics in particular, is normally established in healthy volunteers.

Clinicians, while treating patients in their care, use the information so obtained. Antimicrobials vary considerably in their in vivo bioavailability when administered orally. Dissolution efficiencies of different brands of film-coated ciprofloxacin tablets have been monitored in acetic acid and phosphate buffer, $\mathrm{pH} 7 \cdot 4$, and it has been found that five of the six brands with a $60-70 \%$ fall in active ingredients within $30 \mathrm{~min}$ are adequately bioavailable in vivo. The sixth brand shows a $<40 \%$ drop in ciprofloxacin content and is less bioavailable (Ofoeule et al., 2001). Among sick patients, any poor ciprofloxacin bioavailability would result in therapeutic failures coupled with the inevitable emergence of resistant Salmonella enterica serotype Typhi/Paratyphi isolates.

\section{Subhash C. Arya and Nirmala Agarwal}

Sant Parmanand Hospital, 18 Alipore

Road, Delhi-110054, India

Correspondence: Subhash C. Arya (subhashji@hotmail.com)

Ofoeule, S. I., Udeogaranya, P. O. \& Okonata, J. M. (2001). Prediction of in vivo bioavailability of six brands of ciprofloxacin film coated tablets using the concept of dissolution efficiency (DE). Boll Chim Farm 140, 187-191.

Renuka, K., Sood, S., Das, B. K. \& Kapil, A. (2005). High-level ciprofloxacin resistance in Salmonella enterica serotype Typhi in India. J Med Microbiol 54, 999-1000.

Taylor, R. B., Shakoor, O., Behrens, R. H., Everard, M., Low, A. S., Wangboonskul, J., Reid, R. G. \& Kolawole, J. A. (2001). Pharmacopoeial quality of drugs supplied by Nigerian pharmacies. Lancet 357, 1932-1936. 